\title{
Efeito do uso do xisto em características químicas do solo e nutrição do tomateiro
}

\author{
Hamilton Seron Pereira ${ }^{1,3}$; Godofredo César Vitti ${ }^{2}$ \\ ${ }^{1}$ UFG, 75800-000 Jataí-GO; E-mail: hsp@jatai.ufg.br; ${ }^{2}$ USP/ESALQ, Depto. Solos e Nutrição de Plantas, C. Postal 9, 13418-900 \\ Piracicaba-SP; E-mail: gcvitti@ciagri.usp.br; ${ }^{3}$ Bolsista FAPESP
}

\begin{abstract}
RESUMO
Um dos principais problemas ambientais que ocorre com o processo de exploração do xisto é a produção de quantidades maciças de xisto retortado de alta alcalinidade. Este resíduo, depositado em larga escala, libera elementos tóxicos no ambiente, poluindo o solo, a água de superfície e até o ar atmosférico. Avaliou-se o efeito do xisto nas características químicas do solo e na nutrição do tomateiro em dois experimentos conduzidos em Argissolos Vermelho Amarelo, em ambiente protegido. Foram aplicadas e incorporadas ao solo as doses de $0 ; 3 ; 6 ; 9$ e $12 \mathrm{tha}^{-1}$ de xisto. O delineamento utilizado foi em blocos ao acaso com quatro repetições. A adição de xisto promoveu aumento significativo nos teores de enxofre e silício no solo e nas folhas do tomateiro. Não foram verificadas alterações no valor do $\mathrm{pH}$ do solo nem nos teores de cátions trocáveis, mas, observou-se aumento na disponibilidade de fósforo. Não se verificou dano ao ambiente, por contaminações químicas de metais pesados, nem diminuição na produtividade do tomate em função da aplicação dos tratamentos. O xisto se mostrou apto para ser usado na agricultura, nas doses utilizadas.
\end{abstract}

Palavras-chave: Lycopersicon esculentum, metais pesados, silício.

\begin{abstract}
Effect of the use of schist on chemical characteristics of soil and on tomato nutrition

Among the main environmental problems due to the use of schist is a massive production of highly alkaline schist processing waste. This residue, when deposited in great amounts liberates toxic elements in the atmosphere, polluting the soil, the surface water and the atmospheric air. The effect of the use of schist in agriculture was evaluated on the soil chemical attributes and tomato crop nutrition. Two experiments were performed on Typic Eutrustults under greenhouse conditions. Schist rates of $0 ; 3 ; 6 ; 9$ and $12 \mathrm{tha}^{-1}$ were applied and incorporated in the soil. A randomized block design with four replications was used. Schist use promoted a significant sulfur and silicon content increase in the soil as well as in the tomato leaves; no soil $\mathrm{pH}$ alteration was verified neither the exchangeable cations capacity, but an increase in the phosphorous availability was observed. No environmental damage was verified, such as chemical contamination by heavy metals, nor decrease of the tomato yield. The use of schist as a fertilizer in the evaluated doses is viable in agriculture.
\end{abstract}

Keywords: Lycopersicon esculentum, heavy metals, silicon.

(Recebido para publicação em 25 de outubro de 2002 e aceito em 5 de setembro de 2003)

$\mathrm{D}^{\mathrm{c}}$ etentor da segunda maior reserva de xisto conhecido no mundo e, em função da crise do petróleo nos anos 70 , iniciou-se no Brasil um programa de exploração do xisto na jazida de São Mateus do Sul (PR). A limitação ao desenvolvimento da indústria do xisto deve-se à deposição do resíduo gerado no ambiente (Stark e Redente, 1990). A alta alcalinidade deste resíduo (xisto retortado), com $\mathrm{pH}$ ao redor de 12 , impede severamente os esforços diretos de revegetação (Reddy e Lindsay, 1986). Ainda, o alto teor de sais dificulta o reaproveitamento do xisto, pois podem migrar para a superfície do solo ou concentrar-se na zona radicular, inibindo o restabelecimento das plantas (Fransway e Wagenet, 1981).

Apesar da preocupação com a presença de metais pesados e sais no xisto, observa-se que, dependendo do elemento considerado e do método de utiliza- ção, poderá haver contaminação ou não. É evidente que qualquer outro produto, seja corretivo ou fertilizante, quando aplicado ao solo em quantidades elevadas, poderá também causar danos irreversíveis ao solo e ao ambiente (Schwab et al., 1983; Stark e Redente, 1990).

O xisto retortado tem em sua composição considerável teor de matéria orgânica (15\%) e elevado teor de silício $(52 \%)$ dentre outros elementos. Por isso, apresenta potencial para ser aplicado na agricultura, principalmente na recuperação de áreas degradadas.

$\mathrm{O}$ silício ( $\mathrm{Si}$ ) não é considerado integrante do grupo de elementos essenciais ou funcionais para o crescimento das plantas. No entanto, o crescimento e a produtividade de muitas gramíneas (arroz, cana-de-açúcar, sorgo, milheto, aveia, trigo, milho, grama kikuyu, grama bermuda, etc.) e algumas espécies não gramíneas (alfafa, feijão, tomate, alface e repolho) têm mostrado aumento de produtividade com a maior disponibilidade de Si para as plantas (Elawad e Green, 1979). A sílica dissolvida em solução na forma de $\mathrm{H}_{4} \mathrm{SiO}_{4}$, ocorre em função de um equilíbrio entre a fase sólida e a solução, podendo variar de 1$200 \mathrm{mg} \mathrm{dm}^{-3}$ no solo (Hopps et al., 1977). Devido a esse equilíbrio, a adição de composto solúvel de silício não eleva muito a concentração de $\mathrm{H}_{4} \mathrm{SiO}_{4}$ em solução, já que este passa rapidamente para a fase sólida (Elgawhary e Lindsay, 1972). Os minerais silicatados estão em equilíbrio com o $\mathrm{H}_{4} \mathrm{SiO}_{4}$ em solução e só se solubilizarão quando a concentração na solução do solo se reduzir (Baker e Scrivner, 1985). Desta forma a concentração de sílica em solução será tanto maior quanto for a quantidade de sílica que pode ser solubilizada da fase sólida. 
Avaliou-se a possibilidade da aplicação do xisto na agricultura, verificando seus efeitos na nutrição do tomateiro e nos atributos químicos do solo.

\section{MATERIAL E MÉTODOS}

Para a realização deste trabalho utilizou-se duas áreas. O experimento 1 foi instalado em 1997 no município de Piracicaba (SP) em Argissolo VermelhoAmarelo Eutrófico abrúptico, "A" moderado, textura arenosa/média, com os seguintes atributos químicos: $\mathrm{pH}$ $\left(\mathrm{CaCl}_{2}\right)=5,2$, M.O $=15 \mathrm{~g} \mathrm{~kg}^{-1}$, P (resina) $=111 \mathrm{mg} \mathrm{dm}^{-3}, \mathrm{~K}^{+}=1,7 \mathrm{mmol}_{\mathrm{c}} \mathrm{dm}^{-3}$, $\mathrm{Ca}^{2+}=29 \mathrm{mmol} \mathrm{dm}^{-3}, \mathrm{Mg}^{2+}=14 \mathrm{mmol}$ $\mathrm{dm}^{-3}, \mathrm{H}+\mathrm{Al}=20 \mathrm{mmol}_{\mathrm{c}} \mathrm{dm}^{-3}$. O experimento 2 foi instalado em 1998 no município de Tabapuã (SP), também em Argissolo Vermelho-Amarelo Eutrófico, "A" moderado, textura arenosa/média, com os seguintes atributos químicos: $\mathrm{pH}$ $\left(\mathrm{CaCl}_{2}\right)=5,0$, M.O $=17 \mathrm{~g} \mathrm{~kg}^{-1}$, P (resina) $=90 \mathrm{mg} \mathrm{dm}^{-3}, \mathrm{~K}^{+}=2,8 \mathrm{mmol} \mathrm{dm}^{-3}$, $\mathrm{Ca}^{2+}=28 \mathrm{mmol} \mathrm{dm}^{-3}, \mathrm{Mg}^{2+}=9 \mathrm{mmol}$ $\mathrm{dm}^{-3}, \mathrm{H}+\mathrm{Al}=34 \mathrm{mmol}_{\mathrm{c}} \mathrm{dm}^{-3}$.

Os tratamentos constaram de cinco doses: $0 ; 3 ; 6 ; 9$ e $12 \mathrm{Mg}^{-1}$ de xisto retortado (pós-processamento de retirada do óleo, gás e enxofre) proveniente da Petrobrás-SIX, São Mateus do Sul (PR). Esta doses foram definidas buscando atingir um máximo economicamente viável para o uso do xisto uma vez que não há dados na literatura para defini-las. A análise química do xisto resultou: M.O. $=150 \mathrm{~g} \mathrm{~kg}^{-1}, \mathrm{Si}=247 \mathrm{~g}$ $\mathrm{kg}^{-1}, \mathrm{P}=1,1 \mathrm{~g} \mathrm{~kg}^{-1}, \mathrm{~K}=17 \mathrm{~g} \mathrm{~kg}^{-1}, \mathrm{Ca}=$ $14,5 \mathrm{~g} \mathrm{~kg}^{-1}, \mathrm{Mg}=9,6 \mathrm{~g} \mathrm{~kg}^{-1}, \mathrm{Al}=24,8 \mathrm{~g}$ $\mathrm{kg}^{-1}, \mathrm{Fe}=46,1 \mathrm{~g} \mathrm{~kg}^{-1}, \mathrm{Ti}=457 \mathrm{mg} \mathrm{kg}^{-1}$, $\mathrm{Cu}=60 \mathrm{mg} \mathrm{kg}^{-1}, \mathrm{Zn}=229 \mathrm{mg} \mathrm{kg}^{-1}, \mathrm{Mn}$ $=434 \mathrm{mg} \mathrm{kg}^{-1}, \mathrm{Cr}=55 \mathrm{mg} \mathrm{kg}^{-1}, \mathrm{Mo}=$ $79 \mathrm{mg} \mathrm{kg}^{-1}, \mathrm{Co}=25 \mathrm{mg} \mathrm{kg}^{-1}, \mathrm{Ni}=57$ $\mathrm{mg} \mathrm{kg}^{-1}, \mathrm{~Pb}=109 \mathrm{mg} \mathrm{kg}^{-1}, \mathrm{Ba}=204 \mathrm{mg}$ $\mathrm{kg}^{-1}, \mathrm{Sr}=101 \mathrm{mg} \mathrm{kg}^{-1}, \mathrm{Cd}=4 \mathrm{mg} \mathrm{kg}^{-1}$, $\mathrm{B}=51 \mathrm{mg} \mathrm{kg}^{-1}$ e $\mathrm{Va}=152 \mathrm{mg} \mathrm{kg}^{-1}$.

Os experimentos foram instalados sob cultivo protegido de estrutura metálica na forma de arco com $7 \mathrm{~m}$ de largura por $60 \mathrm{~m}$ de comprimento e altura do vão central com 4,5 m. A cobertura foi com plástico transparente de $150 \mathrm{~mm}$ e as laterais foram fechadas com tela de sombrite preto a $30 \%$. A irrigação utilizada foi do tipo gotejamento com injetor de fertilizante tipo "venturi" para se proceder à fertirrigação com $\mathrm{N}$ e $\mathrm{K}$.
Após o preparo do solo foi realizado o transplante das mudas em linhas duplas com espaçamento de $0,5 \mathrm{~m}$ entre plantas, $0,5 \mathrm{~m}$ na linha dupla e 1,25 $\mathrm{m}$ entre as linhas duplas de plantio. $\mathrm{O}$ delineamento utilizado foi blocos ao acaso com quatro repetições. Cada parcela constou de uma linha dupla de $6 \mathrm{~m}$ de comprimento, perfazendo uma área de $10,5 \mathrm{~m}^{2}$. A adubação básica de plantio foi feita em área total, com $450 \mathrm{~kg}$ ha $^{-1}$ de $\mathrm{P}_{2} \mathrm{O}_{5}$ na forma de superfosfato triplo, antes do plantio e após a aplicação do resíduo, incorporando-se com enxada rotativa a $15 \mathrm{~cm}$. A adubação NK, foi aplicada via fertirrigação, constando de $250 \mathrm{~kg} \mathrm{ha}^{-1}$ de $\mathrm{N}$ (uréia) e 300 $\mathrm{kg} \mathrm{ha}^{-1}$ de $\mathrm{K}_{2} \mathrm{O}(\mathrm{KCl})$, segundo Raij et al. (1992). A fertirrigação teve início uma semana após o transplante, procedendo-se sua aplicação três vezes por semana até o final do experimento.

Além das adubações NPK, foram feitas, semanalmente, após o início da frutificação, adubações foliares com $\mathrm{Ca}$ (1,5 $\left.\mathrm{g} \mathrm{dm}^{-3} \mathrm{de} \mathrm{CaCl}_{2}\right), \mathrm{Mg}\left(0,5 \mathrm{~g} \mathrm{dm}^{-3} \mathrm{de}\right.$ $\mathrm{Mg}$ quelatizado), $\mathrm{B}\left(0,7 \mathrm{~g} \mathrm{dm}^{-3}\right.$ de ácido bórico), e $\mathrm{Mn}\left(0,5 \mathrm{~g} \mathrm{dm}^{-3} \mathrm{de} \mathrm{Mn}\right.$ quelatizado). No experimento 1, as aplicações de Mg e Mn iniciaram-se após o início da colheita, quando se verificou em algumas plantas, sintomas de deficiência destes nutrientes. No experimento 2 estas aplicações ocorreram desde o início das pulverizações.

No período do aparecimento do primeiro fruto maduro, foram realizadas amostragens de folhas para a determinação dos teores de macro e micronutrientes (Malavolta et al., 1997). O silício foi determinado conforme método descrito por Furlani et al. (1978), o qual consiste da extração do Si no material vegetal por fusão da amostra em cadinho de níquel e solubilização com hidróxido de sódio. A determinação foi feita pela formação do complexo BETA-molibdosilicato, reduzido pelo ácido ascórbico ou "azulde-molibdênio", procedendo-se leitura no colorímetro a $810 \mathrm{~nm}$ (Kilmer, 1965). Também foram realizadas análises de metais pesados nos tecidos vegetais $(\mathrm{Ni}$, $\mathrm{Cd}, \mathrm{Cr}, \mathrm{Pb}, \mathrm{Co}, \mathrm{Ti}, \mathrm{Ba}, \mathrm{V}$ e Cu). O Mo também foi analisado. A determinação foi feita em espectrômetro de emissão atômica com plasma de argônio, obten- do-se o extrato com digestão nítricoperclórica de $1 \mathrm{~g}$ do material vegetal e diluindo-se o extrato em $25 \mathrm{~cm}^{3}$ (Malavolta et al., 1997).

Após a última colheita (19 semanas após o transplante), procedeu-se à amostragem de solo na profundidade de $0-10 \mathrm{~cm}$, retirando-se as amostras nas linhas de plantio com o amostrador de solo do tipo "sonda". As amostras de solo foram analisadas quimicamente para os atributos $\mathrm{pH}_{\mathrm{CaCl} 2}, \mathrm{P}, \mathrm{K}^{+}, \mathrm{Ca}^{2+}$, $\mathrm{Mg}^{2+}, \mathrm{H}+\mathrm{Al}$ conforme metodologia de Raij e Quaggio (1983); S-SO confor- $_{4}$ me Vitti (1989); B pelo método da água quente (Berger e Truog, 1939); e Cu, Fe, Mn e Zn, pelo método DTPA (Lindsay e Morvell, 1978). A análise do silício foi efetuada extraindo-se o elemento com oxalato de amônio $0,2 \mathrm{~mol} \mathrm{dm}^{-3}$ a pH 3,0 conforme metodologia descrita por Smith (1984). A determinação foi feita pela formação do complexo BETAmolibdosilicato reduzido pelo ácido ascórbico ou "azul-de-molibdênio", procedendo-se leitura no colorímetro a 810 nm (Kilmer, 1965).

Nas amostras de solo também efetuou-se a análise de Mo e dos metais pesados (Ni, Cd, Cr, Pb, Co, Ti, Ba, V e $\mathrm{Cu}$ ), buscando verificar se o xisto aplicado nas doses usadas causariam alguma alteração na quantidade destes metais. A análise foi realizada por espectrometria de emissão atômica com plasma de argônio e o extrato foi obtido utilizando-se como extrator o Mehlich III (Mehlich, 1984).

A colheita dos frutos iniciou-se por volta da $11^{\mathrm{a}}$ semana após o transplante e procedeu-se duas vezes por semana até a oitava penca de tomate ( 8 a 9 semanas após o início da colheita). Após a obtenção dos resultados, precedeu-se as análises estatísticas de variância dos experimentos com aplicação do teste $\mathrm{F}$ seguida de regressão para avaliar os efeitos das doses de xisto.

\section{RESULTADOS E DISCUSSÃO}

\section{Atributos químicos do solo}

Os dados referentes a $\mathrm{pH}, \mathrm{M} . \mathrm{O} ., \mathrm{Si}$, P, S, K, Ca, Mg, soma de bases, CTC, saturação de bases, B, Cu, Fe, Mn e Zn do solo estão contidos na Tabela 1 . Verifica-se que o xisto não influenciou no 
Tabela 1. Atributos químicos do solo na camada de $0-10 \mathrm{~cm}$ ao final do cultivo do tomateiro (20 semanas após a aplicação do xisto) (metais pesados e Mo - extrator Mehlich III, Si - extraído por oxalato). Piracicaba, ESALQ, 1997.

\begin{tabular}{|c|c|c|c|c|c|c|c|c|c|c|c|c|c|c|}
\hline \multirow{2}{*}{ Tratamentos } & \multirow{2}{*}{$\mathrm{pH}_{\mathrm{CaCl2}}$} & \multirow{2}{*}{$\begin{array}{c}\text { M.O. } \\
\left(\mathrm{g} \mathrm{dm}^{-3}\right)\end{array}$} & \multirow{2}{*}{\multicolumn{2}{|c|}{$\begin{array}{lr}P & S \\
\mathrm{mg} \mathrm{dm}^{-3}\end{array}$}} & $\mathbf{K}$ & $\mathrm{Ca}$ & $\mathrm{Mg}$ & SB & $\mathbf{T}$ & \multirow{3}{*}{ V (\%) } & $\mathrm{Cu}$ & $\mathrm{Fe}$ & Mn & \multirow[t]{2}{*}{$\mathrm{Zn}$} \\
\hline & & & & & \multicolumn{5}{|c|}{ mmolc $\mathrm{dm}^{-3}$} & & \multicolumn{3}{|c|}{$\mathrm{mg} \mathrm{dm}^{-3}$} & \\
\hline \multicolumn{14}{|c|}{ Experimento 1} & \\
\hline Testemunha & 6,3 & 24 & 177 & 23 & 1,6 & 65 & 16 & 82 & 97 & 1,9 & 2,0 & 40 & 11 & 3,5 \\
\hline Xisto $\left(3 \mathrm{t} \mathrm{ha}^{-1}\right)$ & 6,4 & 21 & 177 & 36 & 1,5 & 77 & 19 & 98 & 112 & 1,8 & 2,1 & 40 & 13 & 3,3 \\
\hline Xisto $\left(6 \mathrm{t} \mathrm{ha}^{-1}\right)$ & 6,2 & 25 & 275 & 44 & 1,6 & 68 & 16 & 85 & 100 & 1,8 & 2,3 & 47 & 12 & 3,8 \\
\hline Xisto $\left(9 \mathrm{t} \mathrm{ha}^{-1}\right)$ & 6,4 & 23 & 244 & 64 & 1,6 & 74 & 18 & 93 & 107 & 2,1 & 2,3 & 43 & 14 & 4,2 \\
\hline Xisto $\left(12 \mathrm{t} \mathrm{ha}^{-1}\right)$ & 6,1 & 23 & 252 & 128 & 1,6 & 79 & 21 & 101 & 116 & 1,9 & 2,2 & 44 & 13 & 3,7 \\
\hline C. V. $(\%)$ & 3,1 & 12,2 & 25,2 & 21,5 & 25,9 & 17,4 & 30,8 & 18,7 & 15,9 & 19,5 & 22,7 & 15,4 & 21,0 & 19,8 \\
\hline Valor de $\mathrm{F}$ & $1,2^{\mathrm{NS}}$ & $1,2^{\mathrm{NS}}$ & $2,5^{\mathrm{NS}}$ & $41,5^{* *}$ & $0,1^{\mathrm{NS}}$ & $0,9^{\mathrm{NS}}$ & $0,6^{\mathrm{NS}}$ & $0,9^{\mathrm{NS}}$ & $1,0^{\mathrm{NS}}$ & $0,4^{\mathrm{NS}}$ & $0,3^{\mathrm{NS}}$ & $0,9^{\mathrm{NS}}$ & $0,6^{\mathrm{NS}}$ & $0,7^{\mathrm{NS}}$ \\
\hline \multicolumn{15}{|c|}{ Experimento 2} \\
\hline Testemunha & 5,7 & 21 & 238 & 6 & 1,4 & 60 & 18 & 79 & 100 & 0,7 & 2,8 & 39 & 21 & 6,7 \\
\hline Xisto $\left(3 \mathrm{t} \mathrm{ha}^{-1}\right)$ & 5,6 & 24 & 228 & 26 & 1,8 & 72 & 19 & 92 & 112 & 0,8 & 2,8 & 34 & 23 & 6,7 \\
\hline Xisto $\left(6 \mathrm{t} \mathrm{ha}^{-1}\right)$ & 5,7 & 25 & 239 & 42 & 2,1 & 74 & 22 & 98 & 121 & 0,6 & 3,0 & 38 & 24 & 7,6 \\
\hline Xisto (9 t ha $\left.{ }^{-1}\right)$ & 5,7 & 21 & 297 & 40 & 2,0 & 71 & 21 & 93 & 113 & 0,7 & 3,0 & 42 & 22 & 6,1 \\
\hline Xisto $\left(12 \mathrm{t} \mathrm{ha}^{-1}\right)$ & 5,9 & 23 & 353 & 34 & 1,6 & 78 & 22 & 101 & 120 & 0,7 & 2,6 & 32 & 19 & 6,7 \\
\hline C. V. $(\%)$ & 5,3 & 14,5 & 26,4 & 20,5 & 30,8 & 11,2 & 16,4 & 9,5 & 8,7 & 31,8 & 21,9 & 12,5 & 15,1 & 21,4 \\
\hline \multirow[t]{3}{*}{ Valor de $\mathrm{F}$} & $0,6^{\mathrm{NS}}$ & $1,1^{\mathrm{NS}}$ & $2,3^{\mathrm{NS}}$ & $24,9^{* *}$ & $0,6^{\mathrm{NS}}$ & $2,7^{\mathrm{NS}}$ & $1,6^{\mathrm{NS}}$ & $3,7^{*}$ & $2,8^{\mathrm{NS}}$ & $0,3^{\mathrm{NS}}$ & $0,2^{\mathrm{NS}}$ & $4,5^{*}$ & $1,5^{\mathrm{NS}}$ & $0,7^{\mathrm{NS}}$ \\
\hline & $\mathbf{S i}$ & Mo & & $\mathrm{Ni}$ & $\mathrm{Cd}$ & $\mathrm{Cr}$ & & $\mathrm{Pb}$ & $\mathrm{Ba}$ & $\mathbf{v}$ & $\mathrm{Cu}$ & Co & & $\mathrm{Ti}$ \\
\hline & \multicolumn{14}{|c|}{$\mathrm{mg} \mathrm{kg}^{-1}$} \\
\hline \multicolumn{15}{|c|}{ Experimento 1} \\
\hline Testemunha & 53 & 0,10 & & 0,9 & 0,3 & 0,5 & & 2,2 & 20,4 & 1,6 & 3,9 & 1,0 & & 0,5 \\
\hline Xisto $\left(3 \mathrm{t} \mathrm{ha}^{-1}\right)$ & 58 & 0,10 & & 1,0 & 0,3 & 0,6 & & 2,3 & 19,0 & 1,6 & 3,6 & 1,2 & & 0,5 \\
\hline Xisto $\left(6 \mathrm{t} \mathrm{ha}^{-1}\right)$ & 63 & 0,1 & & 1,5 & 0,3 & 0,7 & & 2,7 & 17,6 & 1,9 & 4,5 & 1,2 & & 0,6 \\
\hline Xisto $\left(9 \mathrm{t} \mathrm{ha}^{-1}\right)$ & 65 & 0,12 & & 1,5 & 0,3 & 0,7 & & 3,0 & 20,4 & 2,0 & 4,1 & 1,3 & & 0,5 \\
\hline Xisto $\left(12 \mathrm{t} \mathrm{ha}^{-1}\right)$ & 69 & 0,1 & & 1,1 & 0,3 & 0,6 & & 2,1 & 16,9 & 1,6 & 4,1 & 1,1 & & 0,5 \\
\hline C. V. $(\%)$ & 27,5 & 7,8 & & 31,3 & 27,0 & - & & 23,1 & 17,9 & 26,6 & 14,5 & 15,3 & & 8,8 \\
\hline Valor de F & $0,5^{\mathrm{NS}}$ & $3,1^{*}$ & & $2,3^{\mathrm{NS}}$ & $0,7^{\mathrm{NS}}$ & - & & $1,7^{\mathrm{NS}}$ & $0,9^{\mathrm{NS}}$ & $0,7^{\mathrm{NS}}$ & $1,3^{\mathrm{NS}}$ & $2,4^{\mathrm{NS}}$ & & $1,0^{\mathrm{NS}}$ \\
\hline \multicolumn{15}{|c|}{ Experimento 2} \\
\hline Testemunha & 84 & 0,04 & & 0,26 & 0,07 & 0,16 & & 2,9 & - & 1,1 & 2,7 & 0,9 & & 0,57 \\
\hline Xisto $\left(3 \mathrm{t} \mathrm{ha}^{-1}\right)$ & 97 & 0,06 & & 0,25 & 0,07 & 0,20 & & 2,8 & - & 1,2 & 2,7 & 0,9 & & 0,57 \\
\hline Xisto $\left(6 \mathrm{t} \mathrm{ha}^{-1}\right)$ & 100 & 0,06 & & 0,26 & 0,08 & 0,17 & & 2,7 & - & 1,2 & 2,5 & 1,0 & & 0,55 \\
\hline Xisto $\left(9 \mathrm{t} \mathrm{ha}^{-1}\right)$ & 101 & 0,07 & & 0,29 & 0,08 & 0,18 & & 2,7 & - & 1,1 & 2,4 & 0,9 & & 0,56 \\
\hline Xisto $\left(12 \mathrm{t} \mathrm{ha}^{-1}\right)$ & 109 & 0,07 & & 0,27 & 0,08 & 0,21 & & 2,8 & - & 1,1 & 2,7 & 1,0 & & 0,59 \\
\hline C. V. $(\%)$ & 12.8 & 33,0 & & 9,9 & 20,9 & 28,9 & & 23,0 & - & 4,1 & 20,3 & 14,4 & & 16,4 \\
\hline Valor de F & $2.1^{*}$ & $1,0^{\mathrm{N}}$ & & $1,1^{\mathrm{NS}}$ & $0,2^{\mathrm{NS}}$ & $0,6^{\mathrm{NS}}$ & & $0,1^{\mathrm{NS}}$ & - & $1,3^{\mathrm{NS}}$ & $0,2^{\mathrm{NS}}$ & $0,2^{\mathrm{NS}}$ & & $0,1^{\mathrm{NS}}$ \\
\hline
\end{tabular}

$\mathrm{pH}$ do solo, mesmo nas doses mais elevadas. As baixas concentrações de $\mathrm{Ca} \mathrm{e}$ $\mathrm{Mg}$ do xisto mostram que há poucos silicatos associados a estes elementos, que por sua vez indica a pouca ação do xisto na reação do solo. Uma elevação nos teores de fósforo em função dos tratamentos foi observada, mas não foi significativa. Essa variação pode ter ocorrido devido à ação dos silicatos contidos no xisto, pois o fosfato e a sílica competem diretamente pelos mesmos sítios de adsorção, embora a força de adsorção do fosfato seja maior que a do $\mathrm{H}_{4} \mathrm{SiO}_{4}$ em $\mathrm{pH}$ ácido (Reifenberg e
Buckwold, 1954), por isso não se deve destacar que o efeito de outros componentes do xisto também estejam agindo. Outros autores (Obihara e Russel, 1972; Smyth e Sanches, 1980) também comentam que a disponibilidade de fósforo pode ser aumentada pela adição de silicatos e obtiveram resultados semelhantes com a adição de escória de siderurgia, devendo-se considerar que este aumento pode ter ocorrido devido à correção do $\mathrm{pH}$ do solo promovido por esta fonte.

Os tratamentos com xisto promoveram aumentos significativos no enxofre do solo, permitindo ajustar as equações: $\mathrm{y}=7,89 \mathrm{x}+11,9\left(\mathrm{r}^{2}=0,83\right)$ para o experimento 1 e $\mathrm{y}=-0,55 \mathrm{x}^{2}+8,88 \mathrm{x}+5,7\left(\mathrm{r}^{2}\right.$ $=0,99)$ para o experimento 2 . A elevação na concentração de enxofre no solo, em função das doses de xisto é justificada pela sua concentração e disponibilidade elevada no resíduo na forma de pirita (Grossi Sad et al., 1984).

Pequenos aumentos nos teores de cálcio e magnésio, refletiram num aumento significativo da soma de bases do experimento 2. Não se verificaram alterações significativas na matéria orgânica do solo, embora o xisto contenha, em sua composição, quantidade ex- 
Tabela 2. Teores de macro, micronutrientes, silício, Mo e metais pesados no limbo foliar do tomateiro. Piracicaba, ESALQ, 1997.

\begin{tabular}{|c|c|c|c|c|c|c|c|c|c|c|c|}
\hline \multirow{2}{*}{ Tratamentos } & $\mathbf{N}$ & $\mathbf{P}$ & $\mathrm{K}$ & $\mathrm{Ca}$ & Mg & $\mathbf{s}$ & B & $\mathrm{Cu}$ & $\mathrm{Fe}$ & Mn & $\mathrm{Zn}$ \\
\hline & \multicolumn{6}{|c|}{$\mathrm{g} \mathrm{kg}^{-1}$} & \multicolumn{5}{|c|}{$\mathrm{mg} \mathrm{kg}^{-1}$} \\
\hline \multicolumn{12}{|c|}{ Experimento 1} \\
\hline Testemunha & 34,6 & 6,5 & 47,9 & 41,5 & 7,8 & 6,8 & 91,0 & 541,3 & 161,5 & 105,0 & 16,3 \\
\hline Xisto (3 t ha-1) & 34,7 & 5,9 & 44,3 & 39,8 & 8,0 & 7,3 & 86,5 & 548,5 & 160,0 & 83,3 & 17,8 \\
\hline Xisto $\left(6 \mathrm{t} \mathrm{ha}^{-1}\right)$ & 32,0 & 5,0 & 40,0 & 38,5 & 7,4 & 7,8 & 81,5 & 600,0 & 158,8 & 102,8 & 16,3 \\
\hline Xisto (9 tha-1) & 33,3 & 5,3 & 46,9 & 42,6 & 7,8 & 8,1 & 80,5 & 534,8 & 168,8 & 100,3 & 15,8 \\
\hline Xisto $\left(12 \mathrm{t} \mathrm{ha}^{-1}\right)$ & 33,6 & 5,3 & 46,9 & 39,8 & 7,6 & 8,3 & 86,5 & 639,0 & 179,8 & 95,0 & 14,5 \\
\hline C. V. $(\%)$ & 4,1 & 18,5 & 13,3 & 13,4 & 9,9 & 7,2 & 10,8 & 14,6 & 30,8 & 14,9 & 14,3 \\
\hline Valor de $\mathrm{F}$ & $2,6 \mathrm{NS}$ & $1,3 \mathrm{NS}$ & $1,1 \mathrm{Ns}$ & $0,4 \mathrm{NS}$ & $0,3^{N S}$ & $4,9^{*}$ & $0,9 \mathrm{NS}$ & $1,2 \mathrm{NS}$ & $0,1^{\mathrm{NS}}$ & $1,4 \mathrm{NS}$ & $1,0 \mathrm{NS}$ \\
\hline \multicolumn{12}{|c|}{ Experimento 2} \\
\hline Testemunha & 40,8 & 4,3 & 29,0 & 36,1 & 4,4 & 6,1 & 429,0 & 1014,8 & 406,0 & 802,5 & 75,8 \\
\hline Xisto (3 t ha-1) & 36,7 & 4,6 & 28,3 & 40,0 & 4,5 & 6,9 & 432,0 & 1064,5 & 431,0 & 875,3 & 83,4 \\
\hline Xisto $\left(6 \mathrm{t} \mathrm{ha}^{-1}\right)$ & 38,3 & 4,7 & 31,5 & 42,6 & 4,6 & 7,6 & 488,5 & 1105,8 & 418,8 & 929,8 & 90,3 \\
\hline Xisto $\left(9\right.$ t ha $\left.^{-1}\right)$ & 35,5 & 4,4 & 31,1 & 38,0 & 4,3 & 7,6 & 401,8 & 1054,8 & 379,5 & 876,0 & 82,8 \\
\hline Xisto $\left(12 \mathrm{t} \mathrm{ha}^{-1}\right)$ & 39,6 & 4,5 & 30,5 & 39,6 & 4,7 & 8,0 & 394,0 & 1021,3 & 482,0 & 836,8 & 73,0 \\
\hline C. V. $(\%)$ & 5,1 & 9,4 & 16,4 & 11,6 & 9,5 & 9,3 & 16,5 & 17,2 & 26,5 & 14,4 & 23,8 \\
\hline Valor de F & $4,7^{*}$ & $0,5 \mathrm{NS}$ & $0,3 \mathrm{NS}$ & $1,1^{\mathrm{NS}}$ & $0,7^{\mathrm{NS}}$ & $4,9^{* *}$ & $1,1^{\mathrm{NS}}$ & $0,2^{N S}$ & $0,5^{\mathrm{NS}}$ & 0,4 NS & $0,5 \mathrm{NS}$ \\
\hline & Si & Mo & $\mathrm{Ni}$ & $\mathrm{Cd}$ & $\mathrm{Cr}$ & $\mathbf{P b}$ & $\mathrm{Ba}$ & $\mathbf{v}$ & $\mathrm{Cu}$ & Co & $\mathrm{Ti}$ \\
\hline & \multicolumn{11}{|c|}{$\mathrm{mg} \mathrm{kg}^{-1}$} \\
\hline \multicolumn{12}{|c|}{ Experimento 1} \\
\hline Testemunha & 1,27 & 1,64 & 0,87 & 1,0 & 9,8 & 2,4 & 15,9 & 5,8 & 598,9 & 1,0 & 8,2 \\
\hline Xisto $\left(3 t^{h} a^{-1}\right)$ & 1,33 & 1,07 & 0,99 & 1,1 & 9,1 & 2,2 & 12,6 & 5,4 & 642,9 & 1,0 & 7,6 \\
\hline Xisto $\left(6 \mathrm{t} \mathrm{ha}^{-1}\right)$ & 1,60 & 1,37 & 1,01 & 1,3 & 11,3 & 2,7 & 10,4 & 7,1 & 659,6 & 1,0 & 10,4 \\
\hline Xisto (9 t ha-1) & 1,60 & 1,17 & 1,13 & 1,1 & 8,5 & 2,5 & 9,6 & 5,9 & 679,2 & 1,0 & 10,9 \\
\hline Xisto $\left(12 \mathrm{t} \mathrm{ha}^{-1}\right)$ & 1,65 & 1,17 & 1,26 & 1,2 & 10,4 & 2,3 & 9,3 & 5,4 & 706,5 & 1,0 & 12,6 \\
\hline C. V. (\%) & 12,0 & 19,9 & 20,6 & 25,0 & 22,8 & 27,8 & 22,9 & 31,4 & 16,5 & - & 18,5 \\
\hline Valor de F & $3,9^{*}$ & 3,1 NS & $1,9 \mathrm{NS}$ & $0,7^{\mathrm{NS}}$ & $0,9 \mathrm{NS}$ & $0,3^{N S}$ & 2,2 NS & $0,5^{N S}$ & $0,6 \mathrm{NS}$ & - & $5,0^{*}$ \\
\hline \multicolumn{12}{|c|}{ Experimento 2} \\
\hline Testemunha & 1,84 & 0,20 & 0,26 & 0,20 & 2,2 & 8,0 & - & 7,8 & 661,9 & 0,46 & 29,2 \\
\hline Xisto $\left(3 \mathrm{t} \mathrm{ha}^{-1}\right)$ & 2,29 & 0,30 & 0,24 & 0,17 & 1,9 & 8,0 & - & 7,5 & 712,5 & 0,48 & 29,3 \\
\hline Xisto $\left(6 \mathrm{t} \mathrm{ha}^{-1}\right)$ & 2,67 & 0,36 & 0,25 & 0,16 & 1,9 & 8,5 & - & 8,1 & 739,4 & 0,52 & 29,6 \\
\hline Xisto (9 t ha-1) & 2,89 & 0,42 & 0,21 & 0,18 & 1,9 & 8,6 & - & 7,3 & 724,5 & 0,47 & 30,3 \\
\hline Xisto (12 t ha-1) & 3,39 & 0,44 & 0,21 & 0,18 & 1,8 & 7,2 & - & 8,1 & 655,9 & 0,47 & 29,1 \\
\hline C. V. $(\%)$ & 12,9 & 35,3 & 24,0 & 18,5 & 22,3 & 19,7 & - & 14,6 & 13,9 & 13,6 & 17,9 \\
\hline Valor de F & $12,2^{* *}$ & $2,6^{N S}$ & $0,6^{\mathrm{NS}}$ & $1,0 \mathrm{NS}$ & $0,4^{\mathrm{NS}}$ & $0,5^{\mathrm{NS}}$ & - & $0,4^{\mathrm{NS}}$ & $0,6^{\mathrm{NS}}$ & $0,6^{\mathrm{NS}}$ & $0,1^{\mathrm{NS}}$ \\
\hline
\end{tabular}

pressiva de matéria orgânica. Também não se verificaram alterações nos teores dos micronutrientes.

Embora o xisto retortado tenha em sua composição silicatos de baixa solubilidade, sua aplicação proporcionou elevação do teor de silício no solo do experimento 2 com o aumento das doses, representada pela equação $\mathrm{y}=1,78 \mathrm{x}$ $+87,4\left(\mathrm{r}^{2}=0,86\right)$.

$\mathrm{Na}$ Tabela 1 também, encontram-se os resultados de analise de metais pesados (Ni, Cd, Cu, Cr, Pb, Ba, V, Co e Ti) e do Mo no solo. Verifica-se, pelos resultados, que não houve aumento significativo nos teores dos elementos anali- sados em todos os tratamentos, com exceção do molibdênio no experimento 1 , onde a utilização de xisto proporcionou elevação significativa nos teores desse elemento. Este aumento era esperado, pois conforme demonstrado por Stark e Redente (1990), nas áreas onde era depositado o xisto retortado as forragens apresentavam alta concentração de Mo. A baixa concentração de metais pesados no xisto, promove a ele condições de ser usado sem causar danos à natureza. Entretanto trabalhos com doses crescentes devem ser conduzidos com o objetivo de se verificar até que ponto pode ser utilizado sem problemas para o meio.
Avaliação nutricional do tomateiro

Na Tabela 2 são apresentados os teores de macro, micronutrientes, silício, Mo e metais pesados no limbo foliar das plantas de tomateiro. Observa-se que não houve alterações significativas nos teores foliares de fósforo, potássio, cálcio, magnésio e dos micronutrientes em função dos tratamentos.

Para o nitrogênio, observou-se redução nos teores foliar em função das doses de xisto no experimento 2. Os teores de enxofre nas folhas aumentaram significativamente em função das doses de xisto nos dois experimentos, permi- 
Tabela 3. Produção final média de tomate, número de frutos (NF) por parcela e peso médio de frutos (PMF). Piracicaba, ESALQ, 1997.

\begin{tabular}{|c|c|c|c|c|c|c|}
\hline \multirow[b]{2}{*}{ Tratamentos } & \multicolumn{3}{|c|}{ Experimento 1} & \multicolumn{3}{|c|}{ Experimento 2} \\
\hline & $\begin{array}{c}\text { Produção } \\
\left(\mathrm{t} \mathrm{ha}^{-1}\right)\end{array}$ & NF & PMF (g) & $\begin{array}{l}\text { Produção } \\
\left(\text { (t ha-1) }^{-1}\right.\end{array}$ & NF & PMF (g) \\
\hline Testemunha & 75,9 & 782,5 & 88,8 & 112,7 & 982,7 & 109,3 \\
\hline Xisto $3 \mathrm{t} \mathrm{ha}^{-1}$ & 101,3 & 982,0 & 102,2 & 113,5 & 1009,4 & 107,3 \\
\hline Xisto $6 \mathrm{t} \mathrm{ha}^{-1}$ & 97,4 & 1035,0 & 91,9 & 111,4 & 969,4 & 109,8 \\
\hline Xisto $9 \mathrm{t} \mathrm{ha}^{-1}$ & 82,5 & 843,0 & 92,9 & 120,1 & 1046,4 & 109,3 \\
\hline Xisto $12 \mathrm{ha}^{-1}$ & 91,1 & 894,9 & 98,6 & 128,3 & 1117,9 & 109,0 \\
\hline C.V. (\%) & 10,7 & 14,5 & 8,7 & 9,5 & 9,0 & 3,6 \\
\hline Valor de F & $3,5^{\mathrm{ns}}$ & 2,4 ns & 1,7 ns & 1,6 ns & $1,7^{\mathrm{ns}}$ & $0,3 \mathrm{~ns}$ \\
\hline
\end{tabular}

tindo ajustar as equações: $\mathrm{y}=0,13 \mathrm{x}+$ $6,9\left(\mathrm{r}^{2}=0,98\right)$ para o experimento 1 e y $=0,15 \mathrm{x}+6,34\left(\mathrm{r}^{2}=0,90\right)$ para o experimento 2. Sendo reflexo de sua maior disponibilidade no solo.

Os resultados analíticos de silício nas amostras de folha, revelam que as aplicações de xisto aumentaram significativamente o teor deste elemento nas folhas, sendo que as doses mais elevadas de xisto foram as que apresentaram maiores teores, principalmente no experimento 2 (Tabela 2 ), representadas pelas equações $\mathrm{y}=0,03 \mathrm{x}$ $+1,28\left(\mathrm{r}^{2}=0,85\right)$ para o experimento 1 e $y=0,12 x+1,87\left(r^{2}=0,86\right)$ para o experimento 2 . Segundo Ponnamperuma (1972), a disponibilidade de Si para as plantas decorre da sua dissolução no solo e a concentração de ácido silícico na solução do solo depende da reação de adsorção dos sesquióxidos que pode ser afetado pelo $\mathrm{pH}$. Dessa forma, a maior concentração de silicatos solúveis no solo deslocou o equilíbrio, aumentando a concentração de $\mathrm{H}_{4} \mathrm{SiO}_{4}$ em solução e, conseqüentemente, na planta.

Quanto aos metais pesados, a principal alteração verificada foi o aumento significativo no teor de titânio, no experimento 1, em função das doses de xisto. Pequenos decréscimos ocorridos nos teores de bário em relação à testemunha (Tabela 2) foram, provavelmente, devido à complexação deste metal pelo sulfato encontrado no xisto, conforme comentado por Chaudry et al. (1977). Para os demais elementos não se encontraram variações significativas, demonstrando pouca influência do xisto na absorção de metais pela planta do tomateiro.

A análise dos dados obtidos na colheita de tomate (Tabela 3), demonstrou que não houve diferenças significativas entre os tratamentos para nenhum dos parâmetros analisados nos dois experimentos. Os resultados indicam que o xisto, nas doses utilizadas, apresenta potencial para uso na agricultura.

\section{LITERATURA CITADA}

BAKER, J.C.; SCRIVNER, C.L. Simulated movement of silicon on Typic Hapludalf. Soil Science, v.139, n.3, p.265-261, 1985.

BERGER, K.C.; TRUOG, E. Boron determination in soils and plants using the quinazizarin reaction. Industrial and Engineering Chemistry, v.11, n.3, p.540-545, 1939.

CHAUDRY, F.M.; WALLACE, A.; MUELLER, R.T. Barium toxicity in plants. Communications in Soil Science and Plant Analysis, v.8, n.8, p.795800, 1977.

ELAWAD, S.H.; GREEN Jr., V.E. Silicon and the rice plant environmental: a review of recent research. Revista IL Riso, v.28, n.2, p.235-253, 1979.

ELGAWHARY, S.M.; LINDSAY, W.L. Solubility of silica in soil. Soil Science Society of American Journal, v.36, n.3, p.430-433, 1972.

FRANSWAY, D.F.; WAGENET, R.J. Salt release and movement in process oil shale. Journal Environmental Quality, v.10, n.1, p.107-113, 1981. FURLANI, P.R.; GALLO, J.R. Determinação de silício em material vegetal pelo método colorimétrico do "azul-de-molibdênio". Bragantia, Campinas, v.37, n.1, p.5-11, 1978. GROSSI SAD, J.H.; SARAIVA, N.T.A.; PINTO, C.P. Rochas oleígenas da Formação Iratí, área da usina industrial Paraná. Belo Horizonte: GEOSOL V 1, 1984. 95 p. (Relatório final)

HOPPS, H.C.; CARLISLE, E.M.; McKEAGUE, J.A.; SIEVER, R. Van; SOEST, P.J. Silicon. In: MERTZ, W Geochemistry and the Environment. Washington, National Academic Society, 1977. v.2, p. 54-72.

KILMER, V.J. Silicon. In: BLACK, C.A., (Ed.) Methods of soil analysis. Madison: Americam Society Agronomy, 1965. p.959-962. (Agronomy, 9)

LINDSAY, W.L.; MORVELL, W.A. Development of a DTPA soil test for zinc, iron, manganese and copper. Soil Science Society of America Journal. v.42, n.3, p.421-428, 1978.
MALAVOLTA, E; VITTI, G.C.; OLIVEIRA, S.A. Avaliação do estado nutricional das plantas, princípios e aplicações. Piracicaba, POTAFOS, 1997. $201 \mathrm{p}$.

MEHLICH, A. Mehlich 3 soil test extractant: A modification of Mehlich 2 extractant. Communications in Soil Science and Plant Analysis. v.15, n.1, p.1409-1416, 1984.

OBIHARA, C.H.; RUSSEL, E.W. Specific adsorption of silicate and phosphate by soils. The Journal Soil Science, v.23, n.1, p.105-117, 1972. PONNAMPERUMA, D.L. The chemistry of submerged soils. Advance in Agronomy v.24, p.2996, 1972.

RAIJ, B. van; QUAGGIO, J.A. Métodos de análise de solo para fins de fertilidade. Campinas: Instituto Agronômico, 1983. 31 p. (Boletim Técnico, 81).

RAIJ, B. van; SILVA, N.M.; BATAGLIA, O.C.; QUAGGIO, J.A.; HIROCE, R.; CANTARELLA, H.; BELLINAZZI Jr., R.; DECHEN, A.R.; TRANI, P.E. Recomendação de adubação e calagem para o Estado de São Paulo. Campinas, Instituto Agronômico, 1992. 107 p. (Boletim Técnico, 100)

REDDY, K.J.; LINDSAY, W.L. The solubility relationships of calcium and magnesium minerals in processed oil shells. Journal of Environmental Quality, v.15, n.1, p.1-4, 1986.

REIFENBERG, A.; BUCKWOLD, S.J. The release of silica from soil by the orthophosphate anion. The Journal of Soil Science, v.5, n.1, p.105117, 1954.

SCHWAB, A.P.; LINDSAY, W.L.; SMITH, P.J. Elemental contents of plants growing on soilcovered retorted shale. Journal of Environmental Quality, v.12, n.3, p.301-304, 1983.

SMITH, B.F.L. The determination of silicon in ammonium oxalate extracts of soils. Communications in Soil Science and Plant Analysis. v.15, n.3, p.199-204, 1984.

SMYTH, J.J.; SANCHES, P.A. Effects of lime, silicate and phosphorus applications to an oxissol on phosphorus sorption and ion retention. Soil Science Society of American Journal, v.44, n.3, p.500-505, 1980.

STARK, J.M.; REDENTE, E.F. Plant uptake and cycling of trace elements on retorted oil shale disposal piles. Journal of Environmental Quality, v.19, n.3, p.495-501, 1990.

VITTI, G.C. Avaliação e interpretação do enxofre no solo e na planta. Jaboticabal, FUNEP, 1989. 37 p. 